\title{
A Case of Chromoblastomycosis Caused by Fonsecaea pedrosoi Successfully Treated by Oral Itraconazole Together with Terbinafine
}

\author{
Xu-Cheng Shen $\cdot$ Xiang-Nong Dai $\cdot$ Zhi-Min Xie $\cdot$ Ping Li • \\ Sha Lu $\cdot$ Jia-Hao Li $\cdot$ Yi Zhang $\cdot$ Xing-Dong Ye (D)
}

Received: December 16, 2019 / Published online: February 21, 2020

(C) The Author(s) 2020

\begin{abstract}
Patients with chromoblastomycosis (CBM) usually have a history of local skin damage related to outdoor activities, mainly manifested as chronic refractory proliferative pathologic changes. We report a case of a 56-year-old man with CBM, identified as Fonsecaea pedrosoi infection by fungal culture and gene sequencing. This patient was successfully treated with a regimen of oral itraconazole (ITZ) and terbinafine lasting 7 months. Through in vitro drug sensitivity tests, minimum inhibitory concentrations of amphotericin, ITZ, and terbinafine were $1 \mu \mathrm{g} / \mathrm{ml}, \quad 0.25 \mu \mathrm{g} / \mathrm{ml}$, and $1 \mu \mathrm{g} / \mathrm{ml}$,
\end{abstract}

Enhanced Digital Features To view enhanced digital features for this article go to https://doi.org/10.6084/ m9.figshare.11744127.

X.-C. Shen · X.-N. Dai · Z.-M. Xie · P. Li ·

X.-D. Ye $(\varangle)$

Department of Dermatology, Dermatology Institute of Guangzhou Medical University, Guangzhou Medical University, Guangzhou 510182, China e-mail: yxingdong@qq.com

X.-N. Dai · Y. Zhang · X.-D. Ye

Department of Dermatology, Guangzhou Institute of Dermatology, Guangzhou 510095, China

S. Lu · J.-H. Li

Department of Dermatology, Sun Yat-sen Memorial Hospital, Sun Yat-sen University, Guangzhou

510120, China respectively. In this case, terbinafine was found to be more effective than ITZ.

Keywords: Chromoblastomycosis; Fonsecaea pedrosoi; Fungal gene sequencing; In vitro antifungal susceptibility; Terbinafine

\section{Key Summary Points}

A case of a 56-year-old man was reported with chromoblastomycosis at his left ankle for more than 1 year, identified as $F$. pedrosoi infection by fungal culture and gene sequencing

The minimum inhibitory concentrations (MIC) of drug sensitivity testing in vitro were amphotericin $1 \mu \mathrm{g} / \mathrm{ml}$, itraconazole (ITZ) $0.25 \mu \mathrm{g} / \mathrm{ml}$, and terbinafine $1 \mu \mathrm{g} / \mathrm{ml}$, and they were equally effective against the wild $F$. pedrosoi strain. The patient was successfully treated with oral ITZ together with terbinafine

Though the MIC of ITZ was lower than that of the other two antifungal agents, we concluded that the drug sensitivity test result of ITZ was not predictive of clinical results. ITZ seems to have had less of an analgesic effect than terbinafine 


\section{INTRODUCTION}

Chromoblastomycosis (CBM), also known as chromomycosis, is a chronic fungal infection of the skin caused by pathogenic fungi [1]. The most common etiologic agents are Cladophialophora carrionii and Fonsecaea pedrosoi. C. carrionii is found in drier climates, whereas $F$. pedrosoi is seen in humid forests [2]. CMB is one of the most common implantation fungal infections, and it occurs worldwide, particularly in tropical and subtropical regions, such as those of southern China [1-3]. In China, CBM was first described by Yew in 1951 [4] and by other authors afterward $[3,5,6]$. CBM is difficult to cure, especially in patients with moderate to severe cases. Here, we report a case of mild CBM of the plaque type caused by $F$. pedrosoi in China that was successfully treated with oral itraconazole (ITZ) and terbinafine.

\section{CASE PRESENTATION}

\section{Case History}

A 56-year-old man from Hainan, China, visited our clinic with a progressively growing, painful red plaque at the inner side of his left ankle following an injury caused by a dust-covered dry root. He had been injured 18 months earlier during his work as a root carver in Hainan Province, a tropical region in southern China. He had sought treatment at more than one hospital, but the plaque did not improve and gradually enlarged, and its central area began to ulcerate and exudate, with occasional purulent discharge. The patient was otherwise healthy. Medical examination showed the patient's vital signs and nervous system were normal. There was an elliptic red plaque on his left ankle, and he felt pain when it was squeezed. The related lymph nodes were not swollen. Informed consent was obtained from the patient for publication of the article.

\section{Fungal Culture and Identification}

A small piece of tissue was cut from the plaque at the left ankle for mycologic culture and histopathologic examination. Before treatment, this piece was divided into four equal parts and inoculated in four tubes on chloramphenicolcontaining Sabouraud glucose agar (SGA) with or without $0.25 \%$ cycloheximide at $26^{\circ} \mathrm{C}$ or $37^{\circ} \mathrm{C}$ respectively. Two weeks later, colonies with the same morphology were observed in all four tubes, and the best colony growth was observed in the tube of SGA medium with $0.25 \%$ cycloheximide at $26^{\circ} \mathrm{C}$. The base of the colony was dark brown and black, the central part was gray and fluffy, and the fluffy surface was wrinkled and radially distributed (Fig. 1b). Cladosporium-type spore formation was observed after the smear (Fig. 1c).

\section{Histopathologic Examination}

Histopathologic examination revealed mild hyperplasia of the epidermis, collagen hyperplasia in the dermis, and superficial capillary hyperplasia. Inflammatory cells were mainly lymphocytes, infiltrating around the blood vessels, and some lymphocytes had invaded the vessel wall (Fig. 1d). No sclerotic bodies were observed with periodic acid-Schiff (PAS) staining.

\section{Molecular Type Identification}

A yeast genomic DNA isolation kit (Tiangen Biochemical Technology Co., Ltd.) was used to extract and purify DNA according to the manufacturer's instructions at a laboratory in the Wuhan branch of Huada Biotech Corp., Shenzhen. A 637-bp fragment of the 18S rRNA region was amplified with the specific primer pair ITS1/ ITS4: ITS1， 5'-TCCGTAGGTGAACCTGCGG-3'; ITS4, 5'-TCCTCCGCTTATTGATATGC-3'. All PCR steps were carried out according to standard protocol. PCR products were electrophoresed on a $0.1 \%$ agarose gel (Fig. $2 \mathrm{a}$ ), and sequences were analyzed by comparison to the F. pedrosoi KMU3817 strain (NCBI Accession No. 


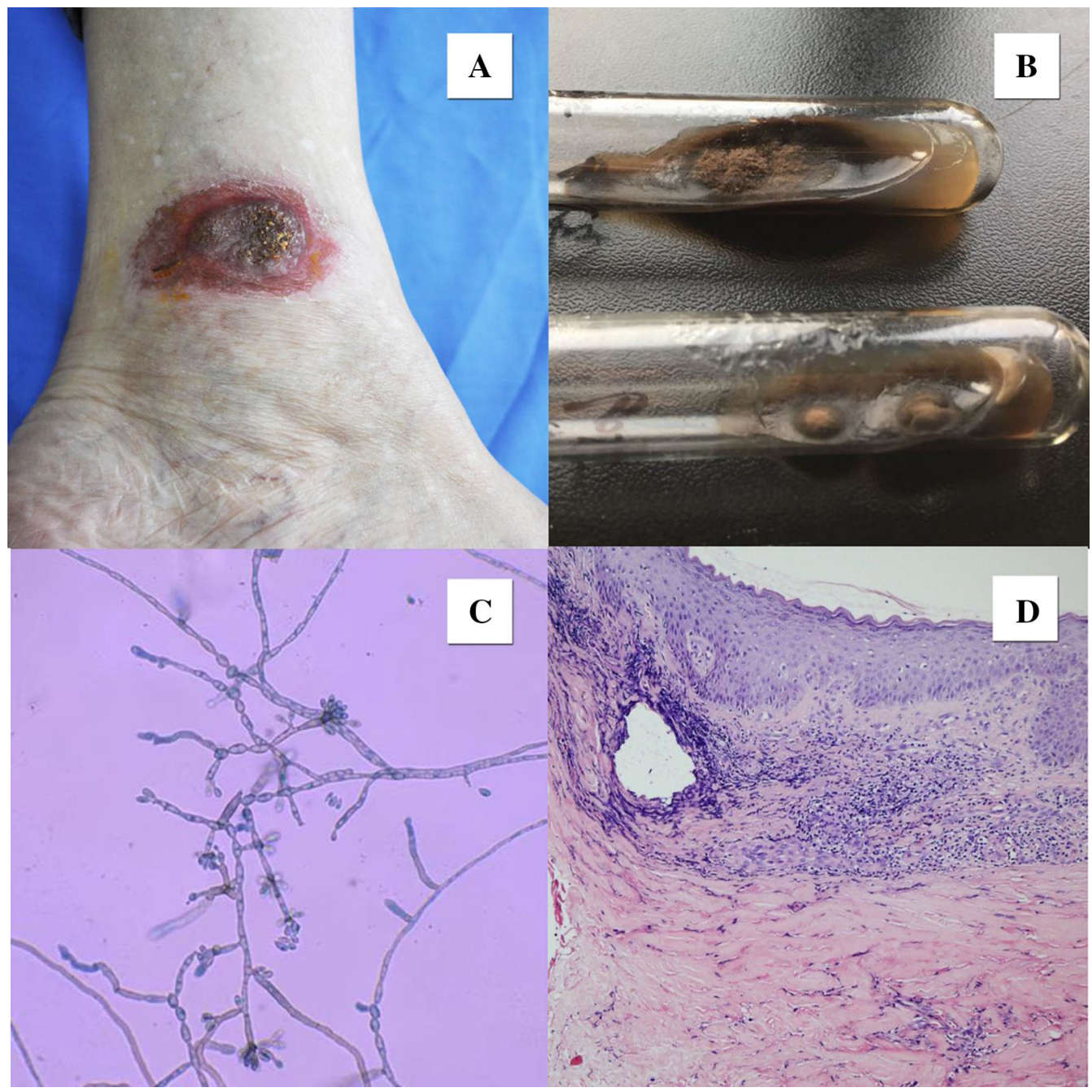

Fig. 1 Data on clinical, mycologic cultures, and histopathologic examination. a An elliptical red plaque approximately $2.5 \mathrm{~cm} \times 4.0 \mathrm{~cm}$ in size with a marginal bulge was visible. $\mathbf{b}$ Black, velvety colonies with raised centers appeared after 4 weeks of incubation at $26^{\circ} \mathrm{C}$ incubator on chloramphenicol-containing SGA with the

AB117982.1) with Bioedit software (Carlsbad, CA, USA) (Fig. 2b, partial sequence).

\section{In Vitro Antifungal Susceptibility}

The wild $F$. pedrosoi strain was cultured in potato dextrose agar medium for the purpose of testing antifungal susceptibility using the diluted micro-broth culture method, and the strain ATCC-22019 of Candida parapsilosis was used for quality control. The minimum inhibitory presence of $0.25 \%$ cycloheximide. c Characteristic conidia or hyphae of $F$. pedrosoi under a microscope. $\mathbf{d}$ Histopathologic examination showing mixed granulomatous inflammation $(\times 100)$, but no micro-abscesses or sclerotic bodies were observed with periodic acid Schiff (PAS) staining

concentrations (MIC) of the tested drugs were obtained as follows: amphotericin $1 \mu \mathrm{g} / \mathrm{ml}$, itraconazole (ITZ) $0.25 \mu \mathrm{g} / \mathrm{ml}$, and terbinafine $1 \mu \mathrm{g} / \mathrm{ml}$.

\section{Therapeutic Intervention and Outcomes}

The patient was initially given ITZ $200 \mathrm{mg}$ once daily, and plaques were significantly less inflamed after 2 weeks of treatment (Fig. 3a, b). ITZ was then administrated for the following 


\section{Marker Sample}

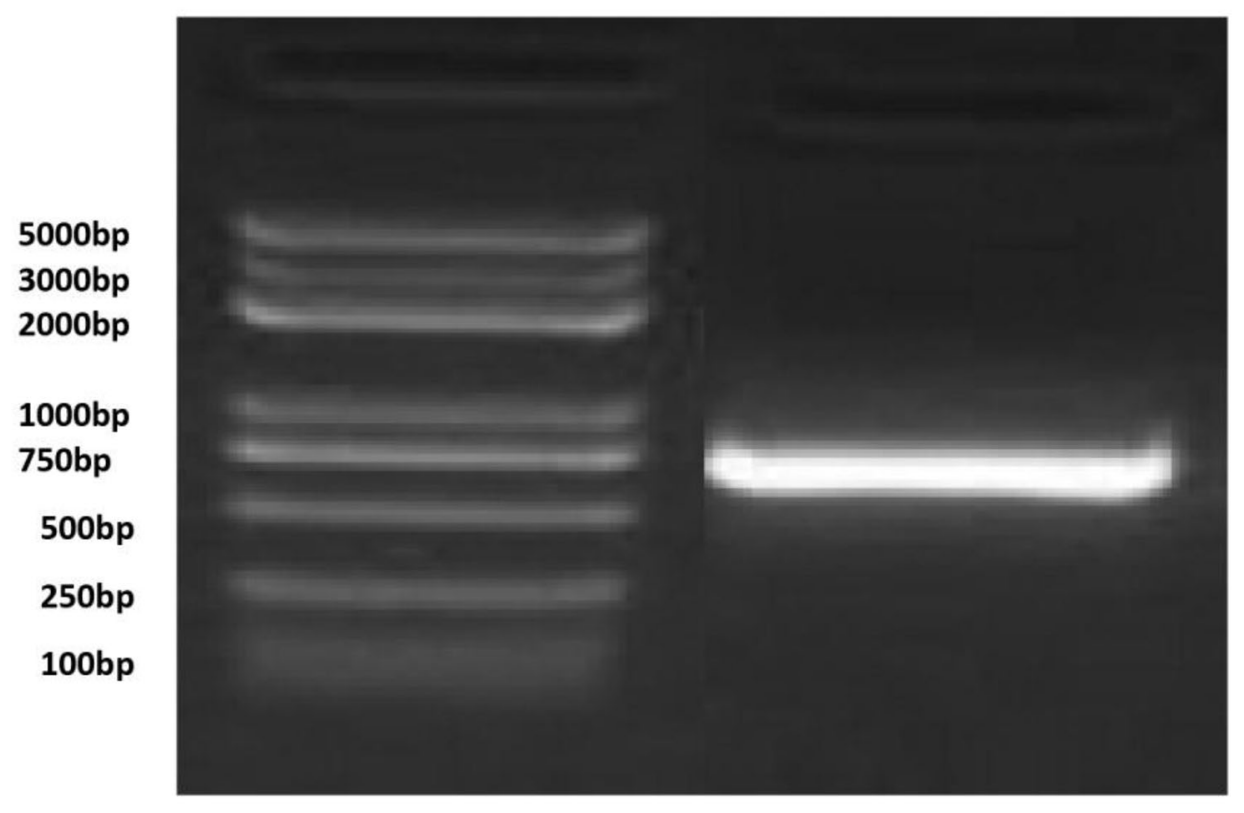

Sample detected.seq FP-AB117982 .1-KMU3817

Sample detected.seq FP-AB117982 . 1-KMU3817

Sample detected.seq FP-AB117982.1-KMU3817

Sample detected.seq FP-AB117982 . 1-KMU3817

Sample detected.seq FP-AB117982.1-KMU3817

Sample detected.seq FP-AB117982.1-KMU3817

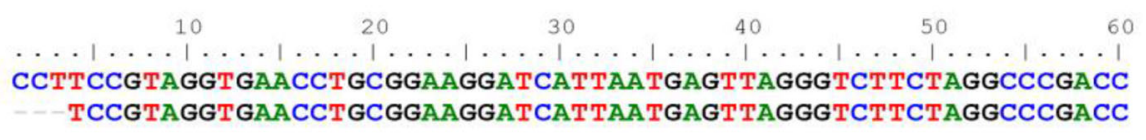

70
$\begin{aligned} & 80 \\ & \text { TCCCAACCCTTTGTTTACTAGACCTCAGTTGCTTCGGCAGGCCCGTCTTAATCTGACCGC }\end{aligned}$
TCCCAACCCTTTGTTTACTAGACCTCAGTTGCTTCGGCAGGCCGTCTTAATCTGACCGC TCCCAACCCTTGTTMCTAGACCICAGTTGCTCGGCAGGCCCGTCTTATCTGACCGC

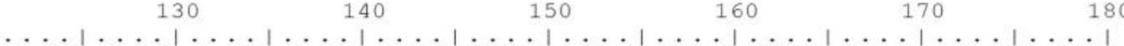
CGGAGGACCGCCTAATACGGGTGTTGCCTCTGGCCAGTGTCTGCCGATAGCCTCATCTAA CGGAGGACCGCCTAATACGGGTGTTGCCTCTGGCCAGTGTCTGCCGATAGCCTCATCTAA 200
CTCTGGATCAATCATGATTACAATGTCTAAGTGATTTATTCAATTAAAAGCAAAACT

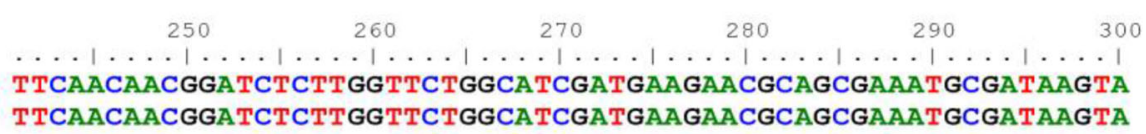

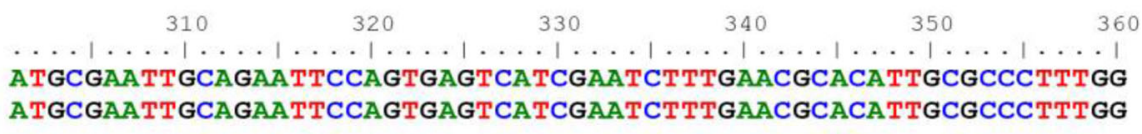

Fig. 2 Data on 1\% agarose gel electrophoresis and blast sequence. a PCR amplification of 637-bp-length target fragment was carried out in a $30 \mu \mathrm{l}$ reaction volume with $0.2 \mu \mathrm{M}$ concentration of each $\mathrm{dNTP}, 0.1 \mu \mathrm{M}$ of each primer, $10 \mathrm{ng}$ of template DNA, and $1.25 \mathrm{U}$ of Taq polymerase with $5 \mathrm{~min}$ at $95^{\circ} \mathrm{C}$, followed by 35 cycles at $95^{\circ} \mathrm{C}$ for $30 \mathrm{~s}, 55^{\circ} \mathrm{C}$ for $30 \mathrm{~s}$, and $72{ }^{\circ} \mathrm{C}$ for $60 \mathrm{~s}$ with a final extension at $72^{\circ} \mathrm{C}$ for $10 \mathrm{~min}, 1 \%$ agarose gel electrophoresis. b Target sample $18 \mathrm{~S}$ rRNA sequence blast with $F$. pedrosoi KMU3817 strain (NCBI Accession No. AB117982.1) 

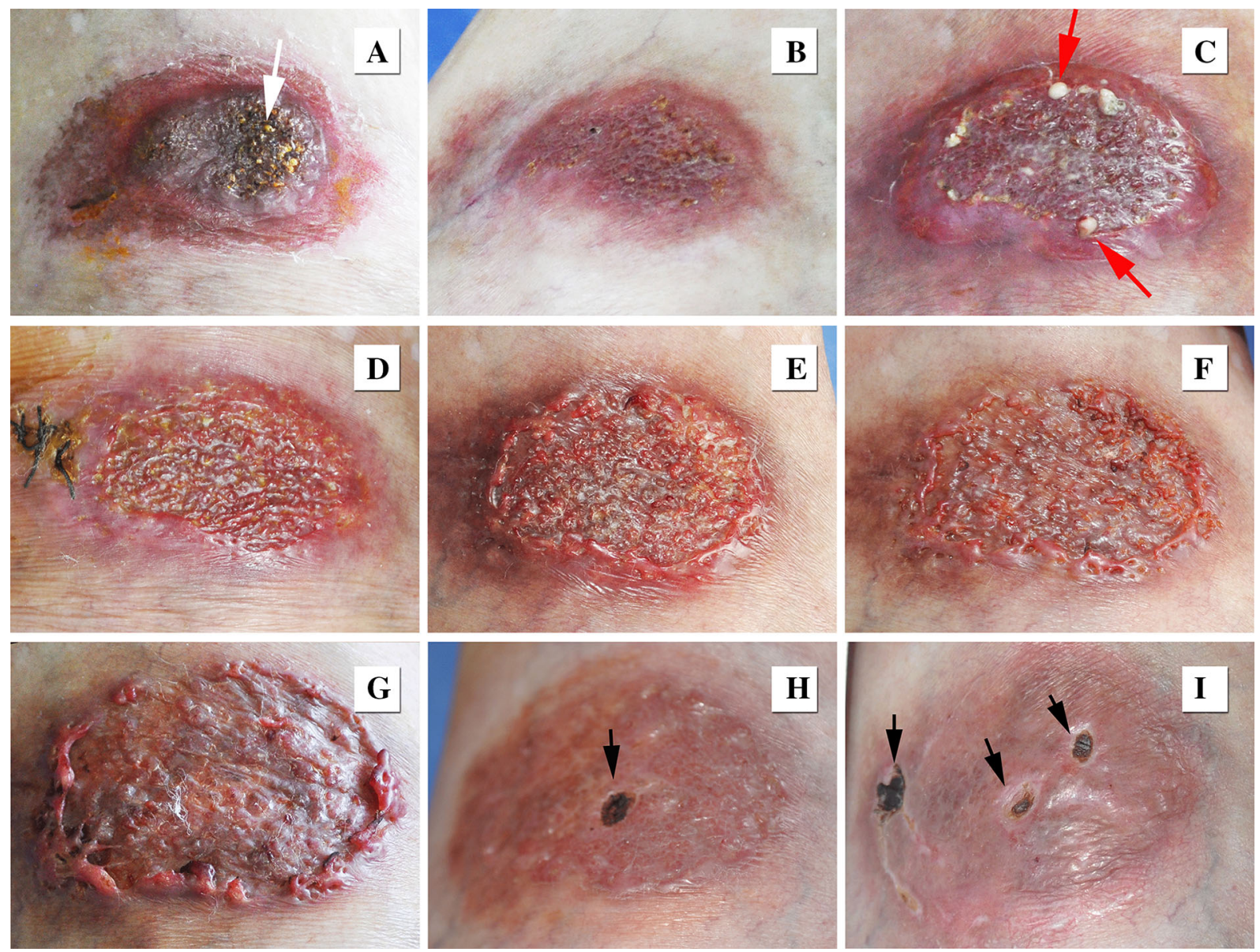

Fig. 3 Group diagram for changes in skin lesions. An elliptical red plaque with marginal bulge surrounded red zone and clustered with necrotic tissue as black dots on the surface of lesions (a, in white arrow). After 8 weeks of treatment with ITZ, the red plaque inflammation had improved significantly (b). At the 12th week, pus-like purulent secretions appeared at the plaque surface after ITZ had been discontinued since 8th week (red arrows)

6 weeks. By the end of the 8th week, the plaque was basically stable, though there was no improvement in pain, and the patient discontinued ITZ himself. At the 12th week, the lesion had gotten worse, and the plaque produced puslike purulent secretions when squeezed (Fig. 3c). The treatment scheme was modified with a combination of terbinafine $250 \mathrm{mg}$ once a day and doxycycline $100 \mathrm{mg}$ twice daily at the 13 th and 14th weeks; the surface purulent discharge improved, and the size of the plaque decreased (Fig. 3d). The terbinafine dose was (c). The plaque had subsided and was flattened, the purulent secretions disappeared, and the lesion showed improvement. The granulation had disappeared, leaving pale red pigmentation when shifted to terbinafine $250 \mathrm{mg}$ to $500 \mathrm{mg}$ daily treatment at the 13th week and afterward $(\mathbf{d}-\mathbf{i})$, and it had crusted over because of applying a solution of poinsettia (a corrosive herbal medicine) to address itching $(\mathbf{h}-\mathbf{i})$

increased from $250 \mathrm{mg}$ to $500 \mathrm{mg}$ daily at the 15 th week and afterward, and the lesion improved continually and finally healed, leaving a pale red scar after 7 months of treatment without side effects (Fig. 3e-i).

\section{DISCUSSION}

CBM actually includes two diseases: chromoblastomycosis, which is caused by a fungus with brown, thick-walled spores in the tissue, 
and phaeohyphomycosis, which is caused by a fungus with brown, separated hyphae in the tissue $[2,7]$. CBM is mainly caused by five species of dark-colored spore fungi belonging to four genera, namely C. carrionii, Fonsecea compacta, F. pedrosioi, Phialophorae verrucosa, and Rhinocladiella cerophilim [1], with the most common etiologic agents being $F$. pedrosoi and C. carrionii. F. pedrosoi is most commonly found in humid forests [2]. Dark spore fungi are found in wet and decaying trees, other plants, and soil, so skin lesions are more common in the hands and feet [7]. The muriform nature of the cells is the main diagnostic feature. These cells may be clustered with necrotic tissue as black dots visible on the surface of lesions. The correct diagnosis is usually made with characteristic histopathology and may be confirmed via culture [2]. In this case, pathologic examination revealed either no characteristic sclerotic bodies suggestive of fungal infections, which may have been due to sectioning, or at most a few spores. Notably, ITZ was effective following drug sensitivity testing, but terbinafine was required as ITZ could neither cure nor provide anti-inflammatory and analgesic effects, indicating that drug susceptibility testing is not always predictive of clinical results.

CBM should be differentiated from verrucous skin tuberculosis, syphilis, disseminated blastomycosis (affected skin), primary cutaneous sporozoites, sporotrichosis, and squamous cell carcinoma. CBM can be divided into five morphologic types: nodular, plaque, tumor, verruciform, and cicatricial [1, 2]. For our patient, a diagnosis of the CBM-plaque type was made, though PAS was negative for brown thick-walled spores in the histopathologic examination.

CBM lesions are often unresponsive to many antifungal and surgical procedures; a complete clinical response is defined as definitive resolution of all lesions [1]. In early stage localized lesions, excision is recommended. Efficacious treatments include physical therapies such as cryotherapy (for lesions under $15 \mathrm{~cm}$ in diameter) [1], photodynamic therapy, and laser treatment $[8,9]$. Topical injection of dextran can be used to treat refractory cases with severe skin lesions [10], in addition to systemic antifungal therapy including terbinafine, ITZ, and amphotericin B [11]. ITZ capsules show clinically significant activity against most CBM agents, although it is more effective against $C$. carrionii than against $F$. pedrosoi $[12,13]$. According to the in vitro drug sensitivity test, amphotericin, ITZ, and terbinafine were equally effective against $F$. pedrosoi, and ITZ was more effective than other agents. Initially, the patient described here was given $200 \mathrm{mg}$ ITZ once daily for 8 weeks, but because it did not alleviate his pain even though the plaque flattened significantly, he discontinued ITZ himself. We then treated him with a terbinafine dose of 250-500 mg once daily to achieve successful treatment. Antifungal susceptibility testing showed the MIC of ITZ to be lower than that of terbinafine, though terbinafine was able to reduce the size of the skin lesions without notable adverse reactions during the treatment.

\section{CONCLUSIONS}

We concluded from drug susceptibility testing that amphotericin, ITZ, and terbinafine are equally effective against $F$. pedroso $i$ in vitro, and the MIC of ITZ was lower than that of the other two agents. However, the drug sensitivity test of ITZ was not predictive of clinical results. ITZ seems to have had less of an analgesic effect than terbinafine.

\section{ACKNOWLEDGEMENTS}

We thank the patient for his participation and we extend our regard to the Guangzhou Institute of Dermatology Research Ethics Committee.

Funding. No funding or sponsorship was received for this study, and The Rapid Service Fee was funded by the authors.

Authorship. All named authors meet the International Committee of Medical Journal Editors (ICMJE) criteria for authorship for this article, take responsibility for the integrity of the work as a whole, and have given their approval for this version to be published. 
Authorship Contributions. Xu-Cheng Shen and Xiang-Nong Dai are co-first authors.

Disclosures. Xu-Cheng Shen, Xiang-Nong Dai, Zhi-Min Xie, Ping Li, Sha Lu, Jia-Hao Li, Yi Zhang, and Xing-Dong Ye have nothing to disclose.

Compliance with Ethics Guidelines. Informed consent was obtained from the patient for publication of the article.

Open Access. This article is licensed under a Creative Commons Attribution-NonCommercial 4.0 International License, which permits any non-commercial use, sharing, adaptation, distribution and reproduction in any medium or format, as long as you give appropriate credit to the original author(s) and the source, provide a link to the Creative Commons licence, and indicate if changes were made. The images or other third party material in this article are included in the article's Creative Commons licence, unless indicated otherwise in a credit line to the material. If material is not included in the article's Creative Commons licence and your intended use is not permitted by statutory regulation or exceeds the permitted use, you will need to obtain permission directly from the copyright holder. To view a copy of this licence, visit http:// creativecommons.org/licenses/by-nc/4.0/.

\section{REFERENCES}

1. Queiroz-Telles F, de Hoog S, Santos DW, et al. Chromoblastomycosis. Clin Microbiol Rev. 2017;30:233-76.

2. Queiroz-Telles F, McGinnis MR, Salkin I, Graybill JR. Subcutaneous mycoses. Infect Dis Clin N Am. 2003;17:59-85.
3. Xi L, Sun J, Lu C, Hoog GS. Molecular diversity of Fonsecaea (Chaetothyriales) causing chromoblastomycosis in Southern China. Med Mycol. 2009;47: 27-33.

4. Cc Yew. Chromoblastomycosis: preliminary report case observed in China. Chin Med J. 1951;69:476.

5. Zhang J, Wu X, Li M, et al. Synergistic effect of terbinafine and amphotericin B in killing Fonsecaea nubica in vitro and in vivo. Rev Inst Med Trop Sao Paulo. 2019;61:e31.

6. Dai W, Chen R, Ren Z. Laboratorial observation and analysis of 287 strains pathogenic Fonsecaea. Chin J Dermatol. 1998;5:8-9.

7. Zhao B, Xu WY, Guo YX, et al. China clinical dermatology. 2nd ed. Nan Jin: Phoenix Publishing Media Group; 2017.

8. Tsianakas A, Pappai D, Basoglu Y, et al. Chromomycosis-successful $\mathrm{CO}_{2}$ laser vaporization. JEADV. 2008;22:1385-6.

9. Huang X, Han K, Wang L, et al. Successful treatment of chromoblastomycosis using ALA-PDT in a patient with leukopenia. Photodiagnosis Photodyn Ther. 2019;26:13-4.

10. Azevedo CM, Marques SG, Resende MA, et al. The use of glucan as immunostimulant in the treatment of a severe case of chromoblastomycosis. Mycoses. 2008;51:341-4.

11. Tyring SK. Tropical dermatology. London: Elsevier Churchill Livingstone; 2006.

12. Najafzadeh MJ, Badali $H$, Illnait-Zaragozi MT, De Hoog GS, Meis JF. In vitro activities of eight antifungal drugs against 55 clinical isolates of Fonsecaea spp. Antimicrob Agents CH. 2010;54:1636-8.

13. Feng $\mathrm{P}$, Najafzadeh $\mathrm{MJ}$, Sun J, et al. In vitro activities of nine antifungal drugs against 81 Phialophora and Cyphellophora isolates. Antimicrob agents $\mathrm{CH}$. 2012;56:6044-7. 